\title{
EL IMPERIO AMENAZADO. LA LITERATURA DE RIDER HAGGARD ANTE LA DECADENCIA
}

\author{
Raquel Sánchez García \\ Universidad Complutense de Madrid
}

\begin{abstract}
RESUMEN: Este trabajo pretende analizar la literatura imperialista inglesa desde la perspectiva de la decadencia del Imperio y la amenaza de los colonizados. Para ello se estudiará la obra de Rider Haggard y en particular una de sus novelas más conocidas y en la que con más claridad se aprecian estas características: She. A History of Adventures (1887). Con esta novela, Haggard creó una metáfora del miedo occidental al mundo primitivo que, pese a que se hallaba sometido a su poder, desafiaba la solidez de la Inglaterra victoriana.
\end{abstract}

PALABRAS CLAVE: Literatura inglesa, gótico imperial, imperialismo, literatura popular, Haggard, decadencia.

\section{THE EMPIRE THREATENED. RIDER HAGGARD'S LITERATURE FACING THE DECADENCE}

\begin{abstract}
This paper analyzes the imperialist English literature from the perspective of the decline of the empire and the threat of the colonized. I have studied the work of Rider Haggard and especially one of his best-known novels that reflects very clearly these features: She. A History of Adventures (1887). With this novel, Haggard created a metaphor of the Western fear to the primitive world, although it was subject to his power: a world that threatened the solidity of Victorian England.

KEYWORDS: English literature, Imperial Gothic, Imperialism, Popular literature, Haggard, decadence.
\end{abstract}

Ridder Haggard (1856-1925) fue uno de los autores victorianos más leídos y más populares. Sus novelas poblaron el imaginario infantil y juvenil de la época con 
evocaciones heroicas acerca de la labor civilizadora de Gran Bretaña en las tierras colonizadas. La mayor parte de sus novelas son relatos de aventuras en los que el protagonista inicia una búsqueda que en principio parece puramente material, pero que en el fondo tiene unos objetivos más próximos a la transformación interior del personaje. Por otra parte, muchas de estas novelas se ambientan en territorio africano, que resulta ser el marco ideal para que el protagonista se enfrente a múltiples desafíos, apartado de las constricciones del mundo victoriano, lejos de los condicionamientos sociales que impiden a los individuos buscar la esencia de lo que son. Los protagonistas de tales aventuras han de superar así una serie de obstáculos impensables en la Gran Bretaña de su tiempo, obstáculos que convertirán la búsqueda africana en un camino iniciático. De este modo, el individuo comienza un viaje físico y metafórico hacia los confines de su propio yo, un yo que sólo es posible encontrar lejos del yo social. Así, mientras que Europa representa la razón y la civilización, África es lo primitivo y, por ende, lo esencial. Las narraciones africanas de Haggard están teñidas, por supuesto, de prejuicios nacionales y raciales, como hombre de su época que fue, sin embargo, el interés de su obra a este respecto merece un análisis más detenido que nos conduce a apreciar más matices de los que aparentemente se observan en una primera lectura de sus novelas.

\section{Haggard en el contexto de la decadencia}

Su obra se enmarca en el contexto del fin de siècle, un momento en el que se extiende por toda Europa un sentimiento de crisis. En este sentido, el concepto de la decadencia se desarrolla a través de otras nociones que acabarán estando estrechamente unidas a él. La principal es la de degeneración. Una degeneración que es a la vez moral y física y que se manifiesta literariamente en figura del vampiro (Warwick, 1998: 202). En el vampiro se personifican las amenazas que reflejan el temor a un mundo cambiante, un mundo en el que el discurso dominante se ve desafiado por nuevas realidades que vienen de la mano de las transformaciones del papel de la mujer en la sociedad, de la visibilidad del otro extraeuropeo y de la aparición de nuevas potencias en el contexto político imperialista. Por otra parte, la decadencia tiene en el individualismo un elemento central (Calinescu, 2003: 172). En un contexto de descomposición de los discursos dominantes y de la pérdida de referentes comunes, la búsqueda individual se generaliza, una búsqueda que en ocasiones acaba desembocando en movimientos que, paradójicamente, abogan por la disolución del yo en aras de la reconstrucción de la vitalidad perdida del ser humano. Otra de las nociones estrechamente ligadas al concepto de decadencia es la de enfermedad. Entendida de múltiples formas, la enfermedad se asocia también a la degeneración. Podría decirse incluso que es una de sus manifestacio- 
nes artísticamente más evidentes. Son muchos los cuadros que muestras esta asociación, particularmente en relación a la sífilis. Podemos recordar aquí la Mors Syphilitica (1905) del pintor belga Félicien Rops. Sin embargo, también se puede aludir a la consunción física que muestra Clorosis, de Sebastià Junyent (1899). Literariamente, como ya se verá en la obra de Haggard y en la de otros contemporáneos, se expresa en forma de lasitud, es decir, de enfermedad del alma que se refleja en el cuerpo, en la materia física que constituye el hombre. En definitiva, la presencia de los conceptos de degeneración y decadencia estuvo tan fuertemente asociada a su expresión artística que, como es sabido, el filósofo Max Nordau dedicó un libro con el nombre de Degeneración (1892) a su análisis. En este contexto finisecular, la obra de Haggard no se halla, ciertamente, en la misma línea que la de Wilde o Huysmans, pero sí muestra el fin de una época y la búsqueda de nuevos valores. En su caso, la búsqueda no comienza en la estética y en el arte, sino en un pasado mitificado y en un misticismo capaz de superar las creencias religiosas establecidas.

\section{Haggard, ¿autor imperialista?}

La mayor parte de la crítica se ha ocupado de enmarcar su obra en la literatura del imperio. Ya en 1967 Alan Sandison lo incluyó en su The Wheel of Empire. A study of the imperial idea in some late nineteenth and early twentieth-century fiction, al lado de Kipling, Conrad y John Buchan. Sandison mantiene una visión positiva acerca de la obra de Haggard, señalando que no hay razones que permitan establecer una conexión directa entre la defensa del imperialismo y su trabajo. Apunta que en las novelas de Haggard se aprecia un cierto relativismo cultural a la hora de enfrentarse a las costumbres de los distintos pueblos que describe, con un convencimiento claro de la superioridad de la civilización occidental, ciertamente, pero sin los componentes de extremo racismo que es posible observar en otros autores. La tendencia a contemplar la obra de Haggard como una creación que va más allá de la literatura infantil se ha visto reforzada desde la década de los sesenta con el florecimiento de los estudios postcoloniales y con los enfoques psicoanalíticos y feministas. De este modo, lo realmente significativo en su obra viene dado por su trascendencia en la cultura y en la mentalidad de su tiempo (Katz, 2010: 4).

Desde este punto de vista es estudiado por Patrick Brantlinger en Rule of Darkness. British Literature and Imperialism, 1830-1914 (1988). En su investigación, Brantlinger se ocupa de analizar el discurso narrativo acerca de la justificación del imperio a través de las obras literarias de diversos autores, así como por medio de 
libros de viajes y textos similares. En el conjunto de autores victorianos impregnados por el espíritu jingoísta, Haggard representa, según Brantlinger, uno de los pocos (al igual que John Buchan) que da una nueva vida a la romántica figura del buen salvaje, especialmente a través de sus dos héroes zulúes Umbopa y Umslopogaas, lo que conduce al lector a contemplar, de la mano del autor, la destrucción de la sociedad zulú por parte de los blancos con menos complacencia de la que suele ser usual en los escritores imperialistas (Brantlinger, 1988: 192). A Brantlinger se debe también la inclusión de Haggard en lo que ha sido denominado Imperial Gothic, género del que se hablará posteriormente.

Más crítica con Haggard se presenta Wendy Katz en su Rider Haggard and the fiction of Empire $(1987,2010)$. Katz apuntala la idea de la escasa calidad estilística de las obras del escritor, pero resalta su trascendencia como fenómeno cultural de su tiempo y lo interpreta como uno de los grandes propagadores de las ideas imperialistas en la Inglaterra victoriana, un propagador consciente y motivado por su misión civilizadora, que se mantuvo firme en su asumido papel como educador de la juventud en los altos ideales del imperio. En la conclusión de su libro resume sus ideas diciendo que "his fiction, only superficially innocuous, contributed generously to the process of shaping the imperial mentality" (Katz, 1987, 2010: 153). Al calificar a Haggard de racista, sexista y antidemócrata, Katz incide en los aspectos más ambiguos de la producción del autor británico. El libro de Katz presenta observaciones de gran interés y pone el énfasis en el uso que Haggard hace de la novela como vehículo de difusión de los ideales imperialistas. Sin embargo, su análisis peca de partidismo mostrando una visión que en algunos aspectos resulta excesivamente unilateral. Es cierto que Haggard hace comentarios racistas, como buena parte de la intelectualidad y de la sociedad británica de la época, pero también ofrece rasgos interesantes de lo que, como se señaló anteriormente, ya apuntaba Sandison en su libro de 1967: en bastantes ocasiones Haggard muestra un relativismo cultural y religioso que sorprende en un hombre de convicciones conservadoras, pues se reconoció como un tory en política.

En esta línea de la crítica postcolonial se encuentran otras obras como Imagining Africa: landscape in H. Rider Haggard's African romances (2001), de Lindy Stiebel, o Rereading the imperial romance: British imperialism and South African resistance in Haggard, Schreiner, and Plaatje, de Laura Chrisman (2000). El primero de estos dos trabajos se ocupa de la construcción romantizada del paisaje africano a través de las obras de nuestro autor, un paisaje que se presenta como referente deseable frente a la turbulenta y hostil vida urbana, un paisaje que remite a la fabulación y a la fantasía que no proporciona la urbanizada Europa. Por su 
parte, la obra de Chrisman se focaliza en el estudio de los tres autores mencionados en el título tratando de discernir las diferencias observables en la forma en que se acercaron literariamente al continente negro en función de sus distintas orientaciones políticas e ideológicas. En este libro, Haggard ocupa la mayor parte del espacio de la autora: cuatro capítulos. Su interpretación insiste en las ambiguas actitudes de Haggard con respecto al proceso de colonización, pues a la vez que lo critica contribuye a su mantenimiento. ${ }^{1}$

Como se acaba de ver, la crítica ha debatido ampliamente acerca de las connotaciones imperialistas de la narración de Haggard. Es innegable, desde luego, que su literatura forma parte de un discurso poblado de evocaciones jingoístas y racistas que le sitúan entre los escritores que aplauden los valores del imperio. Algunas de su obras, incluso, se han convertido en referentes del imaginario imperialista inglés, como King Solomon's Mines. Sin embargo, quedarse sólo ahí nos privaría de los matices que el autor incluye en sus obras y que permiten perfilar más detenidamente su discurso. La mayor parte de la crítica ha apuntado que el conocimiento de primera mano de la realidad africana del que Haggard dispuso es lo que otorga a sus novelas esos matices que singularizan su narración. No hay en Haggard un racismo simple y unilateral, sino la contemplación del Otro en sus múltiples formas. Siempre, por supuesto, desde la atalaya del hombre blanco anglosajón. Antes y después de la publicación de She, Haggard escribió varios artículos y ensayos sobre la situación política en el sur de África y sobre la historia del imperio zulú. El más conocido es Cetywayo and His White Neighbours, publicado en Londres en 1882 y reeditado en 1888. Incluso se ha señalado que es Haggard quien más individualiza a ese otro que es el nativo, haciendo descripciones detenidas de los otros pueblos con un claro interés antropológico (Toda, 2002: 84). Su tratamiento del nativo es, en líneas generales, paternalista.

Por otra parte, desde el momento en que ve a África y a los africanos como Europa y los europeos en el pasado, su idea del imperio es la de una dominación ilustrada (Cohen, 1960: 75-76). Es decir, el presente africano muestra el pasado europeo y, por lo tanto, el segundo forma parte de la identidad del primero: el europeo se contempla en el africano. De este modo, la única concepción posible del imperio es la de un dominio espiritual mediante el que la raza blanca muestre al resto de la humanidad en qué términos puede alcanzarse la superioridad moral.

1. La crítica se ha acercado a este autor desde otras perspectivas, entre las que destacan la biográfica (Cohen, 1960; Beresford Ellis, 1979; Higgins, 1983) y la feminista (Stott, 1989; Murphy, 1999; Young, 2005). 
Para Haggard esto se manifiesta en el indirect rule, que permite el mantenimiento de las costumbres de los pueblos primitivos siempre y cuando no colisionen con los valores morales superiores de la civilización europea. En este sentido, el imperialismo de Haggard se encontraría más próximo a las propuestas de Shepstone que a las agresivas acciones de Cecil Rhodes o Joseph Chamberlain (Etherington, 1984: 104). En mi opinión, hay que tener en cuenta, además, algo que resulta muy significativo para entender su obra: Haggard era políticamente un tory muy crítico con los valores burgueses y comerciales del pueblo inglés, valores que, en su opinión, acabarían con la hegemonía británica en el mundo. Su ideal político consistía en el reforzamiento del mundo rural, de la tierra y de los valores sólidos asociados a ella, por lo que dedicó una buena parte de su tiempo a implicarse en plataformas políticas en pro de la reforma agraria en Inglaterra y a escribir libros sobre este tema como A Farmer's Year, Being His Commonplace Book for 1898 (Londres, 1898), Rural England (Londres, 1902) y The Poor and the Land (Londres, 1905) (Freeman, 2001).

\section{She: metáforas y estereotipos}

She. A History of Adventures es, tal vez, la obra en la que más claramente expone la ambigüedad con la que Haggard trata literariamente las cuestiones relativas al Imperio. Se publicó en 1887, primero en el magacín The Graphic y después en forma de libro. Tuvo una segunda parte, Ayesha. The Return of She, publicada en 1905 y una tercera, que en realidad es una precuela, llamada Wisdom's Daughter: The Life and Love Story of She-Who-Must-Be-Obeyed, aparecida en 1923. Aunque el argumento de la novela es bastante conocido, no está de más hacer un pequeño repaso a la trama, con el objeto de recordar a los personajes y sus peripecias, ya que a lo largo de este trabajo se harán alusiones a ambos.

Horace Holly es un profesor universitario que vive con Leo Vincey, hijo de un amigo suyo ya fallecido. Al cumplir Leo los veinticinco años reciben un mensaje y el fragmento de una antigua cerámica con inscripciones llamada el Sherd of Amenartas. En el mensaje se les conmina a iniciar un viaje a África que tiene relación con la familia de Leo y que, a todas luces, parece descabellado. Sin embargo, deciden emprender la marcha acompañados de su criado Job. Cuando llegan a su destino, se hacen conducir por un guía árabe llamado Mahomed para introducirse en el interior del continente. Llegan al reino de Kôr en el que habita un antiguo pueblo aislado y olvidado del exterior que se denomina a sí mismo la tribu de los "Amahagger". Este pueblo, a diferencia de las poblaciones que les circundan, no es de raza negra, pero tampoco blanca. Se trata de un grupo humano de piel tostada, de cierta belleza y es- 
beltez, pero de gran ferocidad y primitivismo. Los amahagger rinden pleitesía a una reina, mitad bruja, mitad hechicera, a la que jamás han visto ya que siempre se les presenta tapada por velos que ocultan su rostro y su cuerpo. Es conocida por She, Who-Must-Be-Obeyed. Uno de los ancianos de la tribu, Billali, será el encargado por She para ocuparse de los extranjeros hasta que sean recibidos por ella. Mientras tanto, los tres europeos y su guía árabe conocen las costumbres de los amahagger, entre las cuales se encuentra la libertad con la que las mujeres deciden con qué hombre quieren estar. Ustane, una de estas mujeres, se aproxima a Leo y se convierte en su compañera. En ausencia de Billali los amahagger, que son caníbales, deciden realizar un ritual para comerse a Mahomed. Se produce un enfrentamiento en el que Mahomed es asesinado y Leo herido gravemente.

Finalmente, son recibidos por She, quien habita en unas cuevas que habían sido el hogar de la civilización que había ocupado Kôr antes de los amahagger, una civilización desarrollada que había desaparecido hacía miles de años. Lo único que queda de ellos son las momias de sus muertos, que se conservan en perfecto estado. Como Leo se encuentra enfermo, será Holly quien entable los primeros contactos con la reina-hechicera, que aparece velada para evitar que, a causa de su belleza, los hombres se trastornen. Holly, basándose en su condición de hombre de edad al que la belleza de una mujer ya no puede conmover, la convencerá para que se quite el velo en su presencia. A partir del momento en que Holly contempla el rostro de She, su fortaleza racionalista comenzará a derrumbarse. A través de las conversaciones entre ambos, el lector sabrá que el nombre de She es Ayesha y que tiene dos mil años de edad porque ha alcanzado la inmortalidad en el Fuego de la Vida. Su vida consiste en esperar la reencarnación de su antiguo amante, el sacerdote griego Kallikrates, al que ella misma asesinó por haberse fugado con la princesa egipcia Amenartas. ${ }^{2}$ Cuando Ayesha contempla al enfermo Leo, se da cuenta de que se encuentra frente a dicha reencarnación. Sus celos hacia Ustane la conducen, primero, a ordenar su separación de Leo Vincey y, después, a matarla. Recuperado Leo de sus heridas siente que, a pesar de su enfado por la muerte de Ustane, no puede sustraerse a la belleza de Ayesha. Esta última, deseosa de convertir a su reencarnado amante en un ser inmortal, se hace acompañar por los tres europeos hasta las montañas donde se encuentra el Espíritu de la Vida, el fuego de la vida eterna. Una vez allí, Leo muestra muchas reticencias a bañarse en la llama, por lo que Ayesha, tratando de convencerle, entra de nuevo. En este momento, y con una evidente alusión al darwinismo, tiene lugar un proceso de reversión y la bella

2. Esta historia es la que nos contará la propia Ayesha en Wisdom's Daughter. 
e intemporal She pasa por distintos estados físicos, cada vez más deteriorada, y se acaba transformando en un arrugado y viejísimo simio que muere ante los europeos, no sin antes advertir a su amante de que, pese a todo, regresará de nuevo.

La razón por la que She representa, mejor que ninguna otra de sus novelas, la mentalidad del autor estriba en que ofrece un compendio de las ideas de Haggard con respecto al aburguesamiento de la sociedad victoriana, la pérdida de los valores heroicos y la decadencia del imperio, así como un análisis del conjunto de prejuicios y estereotipos de esa misma sociedad. En primer lugar, al repasar la genealogía de Leo Vincey se señala sin ningún rubor que sus antepasados dejaron de tener un comportamiento heroico cuando se dedicaron al comercio, adquiriendo "a dead level of respectability, and a still deader level of mediocrity" (Haggard, 2007: 14). En She el lector encuentra los estereotipos asociados a la raza y a la clase social que es posible hallar en otros autores. Los estereotipos permiten entablar una rápida relación entre el autor y el lector, ya que sitúan a los personajes sin necesidad de realizar grandes matices, sobre todo en este tipo de literatura popular en que los personajes tienden a ser bastante planos. Ciertamente, el representante más elevado de la raza anglosajona, y por lo tanto el héroe, es el personaje de Leo Vincey:

Leo is, with the exception of his hair [tiene el pelo rizado] one of the most English-looking men I ever saw [...] He is very tall and big-chested, and yet not awkward, as so many big men are, and his head is set upon him in such a fashion as to give him a proud and vigorous air, which was well translated in his Amahagger name of the Lion (Haggard, 2007: 257).

Esta descripción física del protagonista responde a la de otros héroes de la novelística de Haggard como Sir Henry Curtis de King Solomon's Mines o Eric Brighteyes, de la obra homónima. El tipo nórdico o anglosajón gustaba a Haggard porque le remitía a su presunto pasado familiar. De hecho, inicia su autobiografía con estas palabras: "There has always been a tradition in my family that we sprang from a certain Sir Andrew Ogard, or Agard, or Haggard [...], a Danish gentleman of the famous Guildenstjerne family whose seat was at Aagaard in Jutland" (Haggard, 2006: 1). Sin embargo, y aunque esto no aparece en She, no se puede dejar de señalar que este estereotipo físico del héroe se traslada en todo menos en el color de la piel a algunos de sus héroes zulúes, y en particular a Umslopogaas, protagonista de Nada the Lily y personaje importante en She and Allan y en Allan Quatermain. Tampoco puede olvidarse que, a pesar de las fantasías nórdicas de Haggard, su auténtico héroe, por el que ha sido conocido y en el que él mismo se encontraba representado, es Allan Quatermain, un hombre maduro, de baja estatura y sin grandes atrac- 
tivos físicos. Así lo dejó escrito en su autobiografía: "I always find it easy to write of Allan Quatermain, who, after all, is only myself set in a variety of imagined situations, thinking my thoughts and looking at life through my eyes" (Haggard, 2006: 228).

El estereotipo vuelve a aparecer en el criado Job, que acompaña a Leo y a Holly en su viaje. Job es descrito como alguien de baja condición social y como tal es tratado todo el tiempo en la novela. Sin embargo, en el territorio de la aventura, en el espacio del imperio, Job, que es inferior socialmente en Inglaterra, se considera superior a los nativos. Job, "a most matter-of-fact specimen of a matter-of-fact class" (Haggard, 2007: 36), simboliza también todos los prejuicios del inglés corriente ante el Otro. Haggard se sirve de este personaje para dos funciones. En primer lugar, para mostrar el lado cómico del contraste cultural (fórmula ya utilizada con el capitán Good en King Solomon's Mines); en segundo lugar, para evidenciar los prejuicios nacionales. Es precisamente en este asunto de los prejuicios donde Haggard muestra una mayor originalidad. Los personajes son situados en un ambiente en el que las costumbres e ideas de la metrópoli se subvierten. Cuando esto no sucede así, al menos queda evidenciada su limitación como instrumentos de convivencia social en un entorno que desconoce esos códigos de conducta, por lo que el individuo, independientemente de que considere primitivas las costumbres a las que se enfrenta, ha de saber reaccionar por su propia supervivencia y la del grupo. En última instancia, en la obra de Haggard eso conduce a una suerte de relativismo que puede ser interpretado como la negación de la universalidad de determinados valores y la aceptación de la diversidad antropológica. Tanto Leo Vincey como Holly se adaptan a lo que ven en el reino de Kôr, pero el criado Job muestra una enorme debilidad ante el Otro, lo que le hace vulnerable al miedo y al conflicto con el nativo: "His nerves -already seriously shaken by what he had undergone since we had arrived in this terrible country- were, as may be imagined, still further disturbed by the spectacle of theses masses of departed humanity" (Haggard, 2007: 260).

En la obra que nos ocupa el lector se encuentra con varias representaciones de la otredad: árabes, negros y, especialmente, el pueblo amahagger. Con respecto a los dos primeros, poco cabe decir porque aparecen como parte de un decorado exótico que circunda la entrada y la salida de los protagonistas del reino de Kôr. Los amahagger, por el contrario, centran la atención de Haggard ya que le sirven como banco de pruebas para su análisis de los comportamientos de otros pueblos y la reacción del europeo ante ellos. Los amahagger son una etnia inventada por Haggard, con piel tostada y rasgos orientales, de gran belleza y primitivismo que habita el espacio anteriormente ocupado por la desarrollada civilización de Kôr. Haggard se explaya en la descripción de sus 
costumbres, entre las que se incluye el canibalismo. En particular, destaca la explicación de sus hábitos sexuales que tanto escandalizan al criado Job y, por ende, a la moral victoriana. Es aquí donde mejor se aprecia esa idea de la convivencia de costumbres y el relativismo moral de Haggard, lo que sorprende en un autor de ideología conservadora. Son muy significativas sus palabras al respecto:

It is very curious to observe how the customs of mankind on this matter vary in different countries, making morality an affair of latitude, and what is right and proper in one place is wrong and improper in another. It must, however, be understood that, as all civilised nations appear to accept it as an axiom that ceremony is the touchstone of morality, there is, even according to our canons, seeing that the interchange of the embrace answers to our ceremony of marriage, which, as we know, justifies most things (Haggard, 2007: 99).

Este relativismo es el que explica que en la literatura de Haggard el lector pueda hallar relaciones amorosas y/o sexuales (nunca explícitas) entre un blanco anglosajón y una mujer nativa, como sucede con Leo Vincey y la amahagger Ustane en She, y con Foulata y el capitán Good en King Solomon's Mines. El interés por la descripción antropológica en Haggard tiene un origen claro en sus experiencias africanas, pero también en su amistad con el antropólogo y folklorista Andrew Lang (Demoor, 1987). Especialista en el estudio de los mitos, Lang aportó a Haggard la idea de que detrás de los aparentemente irracionales elementos que pueden encontrarse en la mitología, se hallan los restos de las narraciones primitivas a partir de las cuales tales mitos surgieron. Además, Lang analizó las doctrinas acerca de la degeneración de los pueblos e impregnó sus conclusiones con una preocupación por el misticismo (Harris, 2003: 179). Morton Cohen ha señalado que ambas cuestiones, la experiencia de Haggard y las conversaciones con Lang, pudieron diseñar los caracteres de la etnia amahagger que aparece en She (Cohen, 1960: 109). Apunta que el referente más próximo puede hallarse en la tribu zulú de los lovedu, que estuvieron gobernados por una reina de piel clara con poderes mágicos llamada Mujaji, mujer que presenta bastantes similitudes con el personaje de Ayesha. ${ }^{3}$

\section{Gótico imperial}

La combinación que es posible encontrar en Haggard entre la aventura africana y el misticismo ha llevado a algunos especialistas a incluirlo en lo que se ha deno-

3. Haggard escribió un artículo sobre esta reina en la African Review, en 1896, que llevó por título "The Death of Majajie". 
minado Imperial Gothic. El gótico imperial fusionaría la ideología imperialista, con sus connotaciones pragmáticas, económicas y científicas, con el interés por lo oculto que aparece ante el europeo cuando se ve inmerso en territorios desconocidos (Brantlinger, 1988: 227). Estaría en directa relación con dos cuestiones que se tratarán en los apartados que siguen: el miedo a la regresión y el miedo a la invasión. La regresión implica una vuelta atrás en la línea evolutiva, temor que nace en la mente del europeo al enfrentarse con los pueblos primitivos que descubre en su conquista del mundo. El miedo a la invasión surge del pánico provocado por la contaminación que puede producir el Otro, el colonizado: el peligro de que llegue a infectar el corazón del imperio. Se trata de un miedo que se produce con la presencia de algún individuo de los espacios coloniales en el entorno doméstico de Gran Bretaña, un individuo (o varios) que se hallan fuera de su lugar natural y que generan disonancia, aprensión y vulnerabilidad. Esa es la percepción que tiene el lector de The Mark of the Beast, de Kipling, o The Sign of Four, de Doyle, e incluso en el caso de novelas de otro género, como The Moonstone, de Wilkie Collins. De este modo, se abre en la mente el europeo una brecha que la confianza en el progreso y en la propia superioridad no puede sellar, una brecha que pone en duda la solidez del mundo construido por medio del desarrollo económico y técnico.

En este contexto, Brantlinger hace referencia a otro aspecto que tiene especial importancia en Haggard, y que ya ha sido mencionado antes: la crisis de los valores tradicionales del pueblo inglés a causa del pragmatismo comercial. En general para todos los autores de este género (entre los que cabe incluir a Stoker, Stevenson, Conrad, Kipling, Lang) existe una clara preocupación por la falta de oportunidades en el mundo moderno para el ejercicio del heroísmo, entendido como un entrenamiento en la búsqueda de la verdad. El imperio y su misión civilizadora aparecen como la barrera de contención ante la barbarie, que sólo es posible activar por medio del reforzamiento de los valores civilizatorios y espirituales. De este modo, algunos de estos autores narran la experiencia colonial en términos épicos, pues el argumento épico, al responder a un género simplificador, permite a la imaginación descubrir en el extranjero las grandes y a la vez simples verdades morales que aparecen desvirtuadas en la metrópoli, que se halla movida únicamente por la consecución del beneficio económico (Brantlinger, 1988: 36).

El otro componente del gótico imperial es el carácter místico o mágico que adquieren algunos elementos que aparecen en la literatura. A través de este mecanismo, los autores entroncan con la tradición gótica de la literatura popular, lo que les permite tener una mayor acogida entre el público, a la vez que ponen en duda, o reflejan, las dudas existentes acerca de la explicación racional del mundo. El fin 
del siglo, con sus problemas existenciales acerca de la validez de los pilares tradicionales de la sociedad, necesitaba de otros referentes que se situaran más allá del materialismo y del cientificismo que pretendía dar sentido al universo humano. Por otra parte, la crisis de las creencias tradicionales y de las religiones establecidas coincidió con el florecimiento de los estudios antropológicos en materia religiosa y con la búsqueda de otras formas de trascendencia. En la literatura se manifestó en unas narrativas llenas de elementos simbólicos y poco o nada realistas.

\section{5. "Going native": la pesadilla de la regresión}

En las descripciones de las costumbres de los amahagger se repiten expresiones como "cold and sullen cruelty", "cannibals", "being hyaenas", "awful and hideous", que revelan su estado de primitivismo, lo que resulta más impactante por la continuada mención a la elevada civilización que ocupaba el lugar en el que viven los amahagger. En la novela se narra un espectáculo, calificado de grotesco, en el que los amahagger danzan con los restos de las momias incendiadas de estos antiguos habitantes. Estos episodios revelan el miedo a la regresión, lo que es conocido en este tipo de literatura como going native. La caída en la abhumnanness y en la degeneración se convierte en la gran pesadilla del europeo, una pesadilla a la vez repulsiva y fascinante (Hurley, 2004: 190). No se trata solamente del interés por lo salvaje, sino de la posibilidad de iniciar un viaje hacia atrás en el proceso de humanización, es decir, la involución. Es necesario considerar el hecho de que Haggard, al igual que otros contemporáneos, vivió y conoció las teorías darwinistas, teorías que si bien él no toma en su sentido literal en sus novelas, sí se traslucen en distintos episodios, y en particular en la desaparición de $\mathrm{She}^{4}$. La convivencia continuada con el nativo, la aceptación de su forma de vida y de sus costumbres y, sobre todo, la inmersión en su mundo irracional y oculto suponen el inicio de la regresión.

En She el lector tiene ante si dos momentos especialmente interesantes a este respecto. Uno de ellos se produce la primera vez que Holly ve a Ayesha envuelta

4. Actualmente resulta difícil imaginar hasta qué punto impactó el darwinismo en la mente de los contemporáneos. Tal vez estas palabras de George Bernard Shaw puedan ayudar a ello: "but if you will remember that Darwin's Origin of Species was published in 1859, you will understand that I belong to a generation which, I think, began life by hoping more from science than perhaps any generation ever hoped before" (Hynes, 1968: 164). Haggard y Shaw nacieron el mismo año: 1856. 
en velos, cita a la que acude acompañado por el jefe amahagger Billali. Ayesha gobierna a los amahagger con dureza y obliga a cualquier individuo que se le acerque a arrodillarse ante ella. Llegado el momento del encuentro, la reacción de Holly es la siguiente: "I halted and felt scared. Indeed, my knees began to give way of their own mere motion; but reflection came to my aid. I was an Englishman, and why, I asked myself, should I creep into the presence of some savage woman as though I were a monkey in fact as well as in name?" (Haggard, 2007: 168). La convicción de la superioridad moral del hombre blanco aún ejerce de freno al camino a la regresión porque su conducta sigue estando dirigida por la racionalidad occidental. Sin embargo, cuando ambos, Leo y Holly, han visto la belleza de Ayesha y ésta ha comenzado a desplegar todo su poder, se muestra la fragilidad de la razón científica y el poder de lo que no es controlable actúa sobre ellos de forma inexorable. Aquí es donde el lector encuentra el segundo momento especialmente significativo al respecto. Ustane, la amante de Leo, ha sido asesinada por She, y Leo no ha reaccionado. Estas son las palabras que le dirige a Holly:

\begin{abstract}
"What am I to do, old fellow?", he groaned, resting his head against my shoulder in the extremity of his grief. "I let her be killed -not that I could help that, but within five minutes I was kissing her murdress over her body. I am a degraded brute, but I cannot resist that" (and here his voice sank) - "that awful sorceress. I know I shall do it again tomorrow; I know that I am in her power for always" (Haggard, 2007: 292).
\end{abstract}

Se trata de la pérdida de la voluntad que vemos también en The Heart of Darkness, no por la violencia, sino por un dejarse llevar, atrapado por una desidia que inmoviliza la firmeza del hombre blanco, que en la obra de Conrad se manifiesta en el calor y la humedad, y que en She se personifica en la seducción que ejerce la belleza todopoderosa de su protagonista femenina. La tensión y la fuerza que exige la civilización occidental se convierte en laxitud en los espacios coloniales y la energía del hombre blanco desaparece, comenzando así su proceso de degeneración.

Sin embargo, y a tenor de las observaciones de algunos críticos (Brantlinger en particular), el native también puede ser blanco y vivir en Gran Bretaña. El obrero revolucionario, el feniano o el lumpen social forman parte del native que habita los bajos fondos de la civilización victoriana. Movidos por fuerzas irracionales, sus comportamientos son imprevisibles. Incluso más, pues el native puede hallarse en cada ser humano. El doctor Jekyll is going native cuando se transforma en Hyde (Brantlinger, 1988: 233). De esta forma, aparece lo irracional dentro del propio individuo; en el seno de su propia sociedad está la expresión de la regresión. El individuo burgués convive con lo primitivo, con lo que él ha sido y puede volver a ser. 
La angustia por la regresión alcanza su más acabada metáfora en la novela en la desaparición de Ayesha. Aquí ya no se trata sólo del going native que afecta sólo a los occidentales, sino de lo que podríamos llamar la pesadilla darwinista de la involución que inquieta a toda la humanidad. En gran medida supone el fracaso de la idea de progreso continuado que había regido a la Europa occidental durante los siglos XVIII y XIX. La constatación de que la evolución no ha de ir necesariamente hacia adelante y de que incluso puede revertir el progreso humano está presente en muchos autores de esta época finisecular que ven disolverse las grandes certezas. Haggard plasma estas angustias en una escena que reproduciré aquí, a pesar de su extensión, por el interés que ofrece. Leo, Holly, el criado Job y Ayesha han ido a buscar la llama del Espíritu de la Vida: el gran secreto de la humanidad guardado en África. De nuevo, el viaje iniciático, plagado de dificultades, aunque esta vez para descubrir el mayor enigma. Llegados a su destino, los personajes, fascinados por lo que ven, se detienen con miedo ante la llama. She, que ya se había bañado en el fuego en el pasado, se introduce en él para dar valor a los hombres. Los tres contemplan maravillados el espectáculo hasta que comienza la transformación, descrita de esta forma por Holly:

True enough -I faint even as I write it in the living presence of that terrible recollection- she was shrivelling up; the golden snake that had encircled her gracious form slipped over her hips and to the ground; smaller and smaller she grew; her skin changed colour, and in place of the perfect whiteness of its lustre it turned dirty brown and yellow, like and old piece of whitered parchement. She felt at her head: the delicate hand was nothing but a claw now, a human talon like that of a badly-preserved Egyptian mummy, and then she seemed to realise what kind of change was passing over her, and she shrieked ah, she shrieked!she rolled upon the floor and shrieked!

Smaller she grew, and smaller yet, till she was no larger than a baboon. Now the skin was puckered into a million wrinkles, and on the shapeless face was the stamp of unutterable age. I never saw anything like it; nobody ever saw anything like the frightful age that was graven on that fearful countenance, no bigger now than that of a two-months' child, though the skull remained the same size, or nearly so, and let all men pray to God they never may, if they wish to keep their reason.

At last she lay still, or only feeble moving. She, who but two minutes before she gazed upon us the loveliest, noblest, most splendid woman the world has ever seen, she lay still before us, near the masses of the own dark hair, no larger than a big monkey, and hideous -ah, too hideous for words-. And yet, think of this -at that very moment I thought of it-it was the same woman! (Haggard, 2007: $356)$. 
Ayesha acaba convertida en algo repulsivo, en unos restos que serán tapados por Holly para evitar la mirada que, a la vez que huye de ellos, no puede dejar de contemplarlos. En este momento Holly es un representante de todos los seres humanos, sobrecogido no sólo por lo extraordinario de la escena que acaba de contemplar, sino también porque el carácter abyecto de lo que tiene delante forma parte de sí mismo a la vez que le repugna. De sí mismo como individuo y de sí mismo como especie. "Overcome with the extremity of horror" (Haggard, 2007: 357) es la frase que utiliza Haggard para describir sus sensaciones. Si cubre los restos de Ayesha con un trapo es, precisamente, para evitar el pánico y el asco que le producen con el deseo de olvidar la animalidad del ser humano (Kristeva, 2004: 7-47).

\section{La reversión imperialista}

El miedo a la invasión del Otro revela, más que un temor a la incursión física (a pesar de que la literatura lo muestre metafóricamente así), un absoluto pánico a la pérdida de la identidad, a la descomposición de los valores morales y civilizatorios de occidente frente a una barbarie que adquiere distintos caracteres, pero que se define en líneas generales por el despotismo, la disolución de la individualidad y de la moralidad burguesa. La literatura popular del fin de siglo revela con absoluta claridad ese miedo y lo proyecta de distintas formas, algunas de ellas, como ha señalado Judith Wilt, darán paso a la literatura de ciencia ficción cuando la amenaza ya no provenga sólo de los territorios colonizados (Wilt, 1981: 64). A este respecto, Haggard escribió: "Soon the ancient mystery of Africa will have vanished". Cuando el misterio de los espacios terrestres haya desaparecido, la amenaza vendrá del exterior o del más allá. Haggard fijará sus fronteras, más que en el espacio, en el tiempo, al contrario que otros autores como Wells en The War of the Worlds, publicada en 1898. De este modo, encontramos que entre la década de los ochenta y el periodo previo a la Primera Guerra Mundial se publicaron muchas novelas sobre la invasión tanto desde el exterior (marcianos, vampiros transilvanos, hormigas amazónicas) como desde el pasado (la reina Tera de Bram Stoker), sin excluir amenazas más reales y acuciantes, como la del Imperio Alemán. Algunas de estas novelas tienen un toque fantástico y gótico, otras son de carácter más realista, como The Taking of Dover (1888), de H.F. Lester, The Sack of London in the Great French War of 1901 (1901, anónima) o The Riddle of the Sands, de Erskine Childers (1903). Como señaló S. Hynes en su estudio The Edwardian Turn of Mind, esta reacción tiene su explicación en la explosión que de las represiones y miedos victorianos se produce entre el fin de siglo y los primeros años del siglo XX. Sin embargo, habría que hacer notar que este tipo de literatura es anterior al periodo al que se refieren los especialistas, pues si nos retrotraemos algo más, llegaremos 
hasta The Last Man, libro de Mary Shelley publicado en 1826 en el que se narra la situación de un mundo futuro devastado por una plaga.

En cualquier caso, y en relación a Haggard, la comparación más próxima es la que cabe hacer con Dracula (1897). El conde inicia un viaje hacia el corazón de occidente (Londres) para dominar Inglaterra que viene a ser la encarnación del mundo civilizado. Sus armas son las del misterio y se sustancian en lo irracional, lo antiguo, lo atávico. Su deseo es acabar con una forma de vida. Dracula se publicó a los sesenta años de que la reina Victoria llegara al trono, en un periodo de introspección y comienzo de la decadencia para Gran Bretaña, así como de la amenaza comercial de Alemania. El vampiro encarna así las sombras que pesan sobre el anglosajón, pues el no-muerto personifica, paradójicamente, al más sano y al más fértil de los vivos y cuando el vampiro muerde a sus víctimas, les otorga más energía. De hecho, cuanto más pálido y más envejecido se ve Jonathan Harker, más fuerte se encuentra el conde, que además es capaz de engendrar una gran descendencia de demonios. Drácula, empleando las armas del colonizador imperialista, amenaza con superar a sus maestros y ocupar su espacio (Arata, 1990: 631-639; Arata, 1996: 107-132).

Esta fantasía de la reversión imperialista se repite también en She y en The Return of She, la segunda parte de la serie. En ambas novelas, la poderosa Ayesha explica a los incrédulos occidentales sus planes de conquista del mundo civilizado. Estos, una vez conocida su debilidad ante la perniciosa influencia de lo irracional, contemplan las fragilidades de la civilización y comienzan a preocuparse seriamente por la posibilidad de mantener la luz de la cultura occidental. Detrás de esto se esconde el desprecio de Haggard por los valores puramente materiales que predominaban en su tiempo y el peligro que eso supone, ya que Inglaterra no tiene nada que oponer a esa embestida, perdida como está en la búsqueda del beneficio inmediato y ajena a los principios espirituales que le dieron grandeza en el pasado. Leo y Holly contemplan en She cómo Ayesha gobierna a los amahagger, a los que hace arrastrarse a sus pies y a los que asesina a la menor muestra de desobediencia o incompetencia. Sin embargo, ella misma señala que "therefore it is [su gobierno] not by force. It is by terror. My empire is of the imagination" (Haggard, 2007: 212). No la violencia, sino el miedo a ella es lo que mantiene las conductas reprimidas en las llanuras de Kôr. El tratamiento del amahagger como alguien inferior a un ser humano es lo que convierte al reino de Ayesha en un reino despótico, lo que hace sacudir las mentes de los dos británicos imbuidos por la concepción whig de la historia, según la cual todo inglés es un hombre nacido libre. Ayesha no se reprime en ningún momento en explayarse en la descripción de su despotismo señalando, a propósito del asesinato de Ustane por sus 
propias manos, que la única razón que ha tenido para hacerlo es que "her sin is that she stands between me and my desire" (Haggard, 2007: 244). Ante el escándalo de Holly, Ayesha desarrolla toda una argumentación basada en el relativismo moral que horroriza a los hombres blancos, una ética que presenta un universo darwinista sin dios (Etherington, 1991: xxviii) que se halla en el trasfondo de los miedos victorianos:

Is it, then, a crime, oh foolish man, to put away that which stands between us and our ends? Then is our life one long crime, my Holly; for day by day we destroy that we may live, since in this world none save the strongest can endure. Those who are weak must perish; the earth is to the strong, and the fruits thereof [...] Thou sayest, too, that a crime breeds evil, but therein thou dost lack experience; for out of crimes come many good things, and out of good grows much evil. Man doeth this and doeth that from the good or evil of his heart; but he knoweth not to what end his moral sense doth prompt him; for when he striketh he is blind to where the blow shall fall, nor can he count the airy threads that weave the web of circumstance. Good and evil, love and hate, night and day, sweet and bitter, man and woman, heaven above and the earth beneath -all these things are necessary, one to the other, and who knows the end of each? I tell thee that there is a hand of Fate that twines them up to bear the burden of its purpose, and all things are gathered in that great rope to which all things are needful. Therefore doth it not become is to say this thing is evil and this good, or the dark is hateful and the light lovely; for to other eyes than ours the evil may be the darkness more beautiful than the day, or all alike be fair (Haggard, 2007: 246-247).

Este largo texto puede ser interpretado no sólo como la exposición de una concepción amoral de la conducta, sino también como expresión de las propias ideas de Haggard (que manifestó en otras ocasiones) acerca de lo incomprensible del destino humano, de la imposibilidad de entender los elementos que rigen el destino del hombre. Sin embargo, también es representación de la quiebra de las creencias ortodoxas acerca de la explicación del mundo proporcionada por la religión. El impacto brutal que tuvo el darwinismo sobre las interpretaciones del pasado y de la trascendencia suministradas por las religiones tradicionales contribuyó a la búsqueda de otros caminos.

En cualquier caso, y para la cuestión que ahora se está tratando, resulta interesante rescatar una conversación que mantienen Ayesha y los dos ingleses, una vez que esta se siente más poderosa por haber logrado su objetivo tras dos mil años de búsqueda. Intrigada por el mundo del que proceden los extranjeros y, en especial por sus formas de gobierno, se mofa de que Gran Bretaña esté regida por una reina que "was venerated and beloved by all right-thinking people in her vast realms" (Haggard, 2007: 309), según le anuncia Holly, quien además le explica que en su 
país el poder descansa en las manos del pueblo. La respuesta de Ayesha no ofrece lugar a dudas sobre sus posicionamientos políticos: "a democracy then surely there is a tyrant, for I have long since seen that democracies, having no clear will of their own, in the end set up a tyrant, and worship him" (Haggard, 2007: 309). Estas palabras, que han servido para que algún crítico califique a Ayesha, un tanto anacrónicamente, de fascista (Etherington, 1984: 46), revelan no sólo las palabras de una reina exótica en el corazón de África sino, una vez más, un estado de opinión entre ciertos sectores de la intelectualidad europea que, desconfiando de la evolución del sistema político liberal y temiendo a la naciente sociedad de masas, optaron por soluciones radicales que alcanzarían su máxima expresión en el siglo $\mathrm{XX}$. Estas desconfianzas hacia la democracia ya se hallan presentes en The Coming Race, de Bulwer-Lytton, e incluso en el propio Dracula. La búsqueda del hombre o de la raza superior, que se sitúan por encima de las mediocridades de la clase burguesa, y el deseo de encontrar otros valores que no sean los del materialismo imperante, de plena raíz nietzscheana, ambientaban la reflexión política e intelectual en el fin de siglo. En el caso de Haggard, no puede decirse que sus posicionamientos políticos defendieran a un líder de tales características, pero lo que sí es obvio es que, como clasista y tory que era, desconfiaba del sufragio universal y consideraba que la extensión del voto a las clases trabajadoras podía suponer un peligro social y que la tiranía era el precio a pagar por la democracia. Haggard era más partidario de una sociedad al estilo tradicional, con las estratificaciones sociales propias del mundo agrario y conservador, por lo que sus ideas políticas se hallaban más cerca de la tradición que de una modernidad en forma de tiranía o en forma de democracia.

Los planes de Ayesha de marchar a Inglaterra con su amado Kalíkrates e imponer su ley hacen temblar a Holly acerca de los resultados de tal proyecto: "In the end she would, I had little doubt, assume absolute rule over the British dominions, and probably over the whole earth, and, though I was sure that she would speedly make ours the most glorious and prosperous empire that the World has ever seen, it would be at the cost of a terrible sacrifice of life" (Haggard, 2007: 310). La regresión final de She no es obstáculo para sus propósitos, pues en The Return of She (1905), la segunda parte de la serie, Haggard hace reaparecer a una Ayesha un tanto transformada pero con los mismos deseos de poder, y esta vez con unos planes mejor trazados que revelan otra de las manifestaciones de esa reversión imperialista:

She took a map of the eastern hemisphere which I had drawn and, placing her finger upon Pekin, said - "There is the place that shall be our home for some few centuries, say three, or five, or seven, should it take so long to shape this people to my liking and our purposes. I have chosen these Chinese because thou tellest 
me that their numbers are uncountable, that they are brave, subtle, and patient, and though now powerless because ill-ruled and untaught, able with their multitudes to flood the little western nations" (Haggard, 1905: 196-197).

La idea de la expansión de una plaga que aplasta al mundo occidental (es decir, a la civilización) es especialmente importante, porque otorga un grado mayor a la fantasía de la invasión, convirtiendo a los invasores en uno de los viejos mitos de la historia europea: la enfermedad, las grandes epidemias que asolaron el continente a lo largo de los siglos y que venían, más o menos figuradamente, del este. El miedo a la invasión no resulta tan creíble si el protagonista es el africano, a quien un imperialista inglés ve como una especie de buen salvaje, por lo que en el caso de She la amenaza es más simbólica que real, pero sí resulta del todo inquietante si el enemigo es el oriental, que procede de un entorno civilizatorio superior. A este respecto, y por curiosidad, resulta interesante indicar que a Haggard no le gustaban los chinos y mostró públicamente su disgusto por la presencia de estos en las minas sudafricanas, por lo que recomendó al gobierno inglés que facilitara la emigración de británicos a este territorio (Etherington, 1984: 95).

\section{Conclusión}

A la luz de las observaciones contenidas en las páginas que preceden a esta conclusión, es posible afirmar que She es el resultado de un ambiente, de una preocupación por el fin de una época. Haggard, inmerso en el pensamiento de su tiempo, mantenía un gran orgullo por los logros de su país, pero a la vez estaba convencido de que el dominio británico en el mundo estaba llegando a su fin. Como muchos contemporáneos, creía que la única forma de salvar la civilización anglosajona era ceder el testigo a los norteamericanos (Bell, 2006). Por otra parte, en la novela se reflejan también otro tipo de miedos: los que le produce el mundo primitivo. Un mundo primitivo del que el hombre europeo no puede sustraerse porque forma parte de su misma esencia: es su pasado. La mirada que proyecta Haggard hacia el África desconocida o colonizada no es la de un europeo soberbio y arrogante, sino la del ser humano que, creyendo superadas sus limitaciones y sus instintos, se encuentra de nuevo con ellos más allá de los engaños que produce la cómoda sociedad burguesa. De este modo, la decadencia adquiere para Haggard un doble componente: el político y el humano. La decadencia es la amenaza de volver a ser lo que se fue: un salvaje. Sin embargo, la decadencia también ofrece la oportunidad de regresar a los viejos valores heroicos que permitieron construir una civilización pervertida en la Inglaterra victoriana por los intereses más puramente materiales. 


\section{Bibliografía}

ARATA, S. (1990). "The Occidental tourist: Dracula and the anxiety of reverse colonization". Victorian Studies 33 (4): 621-645.

ARATA, S. (1996). Fiction of loss in Victorian fin de siècle. Cambridge: Cambridge University Press.

BELL, D. (2006). "From Ancient to Modern in Victorian Imperial Thought". The Historical Journal 49 (3): 735-759.

BERESFORD ELLIS, P. (1978). H. Rider Haggard. A Voice from the Infinite. Londres: Routledge and Kegan Paul.

BRANTLINGER, P. (1988). Rule of Darkness. British Literature and Imperialism, 1830-1914. Londres-Ithaca: Cornell University Press.

CALINESCU, M. (2003). Cinco caras de la modernidad. Modernismo, vanguardia, decadencia, kitsch, postmodernismo. Madrid: Tecnos.

COHEN, M. (1960). Rider Haggard. His life and works. Londres: Hutchison of London.

CHRISMAN, L. (2000). Rereading the imperial romance: British imperialism and South African resistance in Haggard, Schreiner, and Plaatje. Oxford: Clarendon Press; New York: Oxford University Press.

DEMOOR, M. (1987). "Andrew Lang's Letters to H. Rider Haggard: the record of a harmonius friendship". Études Anglaises 40 (3): 313-321.

ETHERINGTON, N. (1978). "Rider Haggard, Imperialism and the Layered Personality". Victorian Studies 22 (1): 71-87.

ETHERINGTON, N. (1984). Rider Haggard. Boston: Twayne Publishers.

ETHERINGTON, N. (1991): "Introduction and notes" en The annotated She. A Critical Edition of H. Rider Haggard's Victorian Romance. Bloomington e Indianápolis: Indiana University Press.

FREEMAN, M. (2001). "Rider Haggard and Rural England: methods of social enquiry in the English countryside: methods of social enquiry in the English countryside". Social history 26 (2): 209-216.

HAGGARD, H. R. (1905). Ayesha: The Return of She. <http://www.gutenberg. org/ebooks/5228>.

HAGGARD, H. R. 2007 (1887). She. A History of Adventures. Londres: Penguin Classics.

HAGGARD, H. R. (1923). Wisdom's Daughter: The Life and Love Story of SheWho-Must-Be-Obeyed. <http://gutenberg.net.au/ebooks02/0200181.txt>.

HAGGARD, H. R. 2006 (1926). The Days of My Life. An Autobiography. Teddington: The Eco Library. 
HARRIS, M. (2003). El desarrollo de la teoría antropológica: historia de las teorías de la cultura. Madrid: Siglo XXI.

HIGGINS, D. S. (1983). Rider Haggard. A Biography. New York: Stein and Day Publishers.

HURLEY, K. (2004). "British Gothic Fiction, 1885-1930" en The Cambridge Companion to Gothic Fiction. (Ed. Jerrold E. Hogle). Cambridge: Cambridge University Press.

HYNES, S. (1968). The Edwardian Turn of Mind. Princeton: Princeton University Press.

KATZ, W. 2010 (1987). Rider Haggard and the Fiction of Empire, Cambridge: Cambridge University Press.

KRISTEVA, J. (2004). "Sobre la abyección” en Poderes de la perversión. México: Siglo XXI.

MURPHY, P. (1999). "The Gendering of History in She", Studies in English Literature, 1500-1900 39 (4): 747-772.

SANDISON, A. (1967). The Wheel of Empire. A study of the imperial idea in some late nineteenth and early twentieth-century fiction. London, Melbourne: Macmillan.

STIEBEL, L. (2001). Imagining Africa: landscape in H. Rider Haggard's African romances. Londres: Greenwood Press.

TODA IGLESIA, $\mathrm{M}^{\mathrm{a}}$ A. (2002). Héroes y amigos: masculinidad, imperialismo y didactismo en la novela de aventuras británica, 1880-1914. Salamanca: Universidad de Salamanca.

STOTT, R. (1989). "The Dark Continent: Africa as Female Body in Haggard's Adventure Fiction". Feminist Review 32: 69-89.

WARWICK, A. (1998). "Vampires and the empire: fears and fictions of the 1890s" en Cultural Politics at the Fin de Siècle. (Ed. S. Ledger y S. McCraken). Cambridge: Cambridge University Press.

WILT, J. (1981). "The Imperial Mouth: Imperialism, the Gothic and Science Fiction”. Journal of Popular Culture 14 (4): 618-628.

YOUNG, S. (2005). "Myths of Castration: Freud's 'Eternal Feminine' and Rider Haggard's She". Victorian Newsletter 108: 21-30. 
